\title{
Consistent behavior of Eulerian Monte Carlo fields at low Reynolds numbers
}

\author{
Luis Valiño*1, Radu Mustata ${ }^{1}$, and Khaled Letaief $^{2}$ \\ ${ }^{1}$ LIFTEC, CSIC - Universidad de Zaragoza, María de Luna 10, 50018 Zaragoza, \\ Spain \\ ${ }^{2}$ ONERA, DEFA/PRA, Chemin de la Hunière, 9112, Palaiseau, France
}

\begin{abstract}
A slightly different version of the Eulerian Monte Carlo method (EMC) (Valio, L., Flow, Turbulence and Combustion, 60:157172, 1998) is presented in this paper. The EMC method is an effective stochastic numerical approach to solve the Probability Density Function (PDF) of reacting species in turbulent flows. In contrast with the original formulation, the spurious Wiener term associated with the molecular diffusion is removed, by splitting the micro-mixing into mean gradient and fluctuating contributions. The evolution of the EMC fields representing the PDF in the proposed formulation is then consistent in the laminar limit: the EMC fields follow the same standard convection-diffusion equation, without any stochastic terms.
\end{abstract}

Keywords: PDF, Monte Carlo, turbulent combustion, partial stochastic differential equations

\section{Introduction}

The one-point probability density function (PDF) of composition [1] is one of the best suited alternatives to calculate turbulent reacting flows. The advantage of the PDF formalism lies on the closed character of the chemical source terms, thus avoiding the modeling of the interaction between sub-grid and resolved scales of these usually highly non linear terms. Closures are however still needed for the scalars turbulent convection and molecular micro-mixing. After appropriate modeling of these two terms, the closed transport equation of the PDF has to be solved. Given the high dimensionality of the problem (space, time and number of scalars), Monte Carlo methods are generally used in order to obtain numerical solutions. Among those methods, the Eulerian Monte Carlo approach (EMC) [2] has proved to be reliable and fast, particularly when used in combination with Large Eddy Simulations [3, 4]. A recent comprehensive review of PDF methods, including EMC, has been made by Haworth [5].

*valino@litec.csic.es 
In EMC formulation, the PDF is described as a set of notional fields, which evolve stochastically with rules deduced from the very PDF transport equation, using either Ito [2] or Stratonovich calculus [6]. One of the mean advantages of this formulation over the Lagrangian approach is that pure Eulerian numerical algorithms can be used to solve the (stochastic) partial differential equations of the notional fields. Such algorithms are employed to obtain the needed turbulent viscosity coefficient from auxiliary $k-\varepsilon$ equations [7], although stochasticity introduces some changes in the implementation.

The whole EMC formulation is deduced for a high Reynolds number flow. Durbin and Petterson state about turbulent models [8]: "They are developed for fully turbulent conditions... however, most transport equation models do converge to a laminar solution...". An example of this is precisely given by the estimation of the turbulent diffusion coefficient $\Gamma$ by $k-\varepsilon$ models, which tends to zero as the flow becomes laminar. Unfortunately, in the original stochastic EMC formulation there are some small inconsistencies in the laminar limit. The purpose of this paper is to use a slightly different derivation in order to address this issue. To ease the reading of this paper, the above mentioned inconsistencies are pointed out once the alternate stochastic formulation is presented.

\section{Formulation}

A dynamically passive scalar $c(\mathbf{x}, t)$ evolving in a velocity field obeys the equation

$$
\frac{\partial c}{\partial t}+u_{j} \frac{\partial c}{\partial x_{j}}=\frac{\partial}{\partial x_{j}}\left(\gamma \frac{\partial c}{\partial x_{j}}\right)
$$

where $\mathbf{u}(\mathbf{x}, t)$ is a solenoidal velocity field governed by the Navier-Stokes equations and $\gamma$ is the molecular diffusivity of the scalar $c\left(\left[\mathrm{~m}^{2} \mathrm{~s}^{-1}\right]\right)$. The solenoidal character of the velocity field is not really necessary (see the comments at the end of this section), and it is used to ease the comparison with the introductory paper of the EMC field method [2].

If the velocity field is turbulent, it will induce, via convection, an apparent random behavior on the scalar field. A general transport equation for the one-point PDF of the previous scalar, 
$P_{c} \equiv P_{c}(\phi ; \mathbf{x}, t)$ can then be derived for different boundary conditions [9]. In the particular case that the PDF covers the whole probability space, the aforementioned transport equation reads [10]:

$$
\begin{aligned}
\frac{\partial P_{c}}{\partial t} & +U_{i} \frac{\partial P_{c}}{\partial x_{i}}+\frac{\partial}{\partial x_{i}}\left(\left\langle u_{i}^{\prime} \mid c=\phi\right\rangle P_{c}\right) \\
& =-\frac{\partial^{2}}{\partial \phi^{2}}\left[\gamma\left\langle\frac{\partial c}{\partial x_{i}} \frac{\partial c}{\partial x_{i}} \mid c=\phi\right\rangle P_{c}\right]+\frac{\partial}{\partial x_{i}}\left(\gamma \frac{\partial P_{c}}{\partial x_{i}}\right),
\end{aligned}
$$

where $\mathbf{U}$ is the mean velocity and $\left\langle\mathbf{u}^{\prime} \mid c=\phi\right\rangle$ is the expected value of the fluctuating velocity conditional on the scalar taking the value $\phi$. An analogous notation will be used for the scalar mean $(C)$ and its fluctuating part $\left(c^{\prime}\right)$.

Two open terms appear in Equation (2). The first one is the transport due to fluctuating velocity (third term on the left hand side), which resembles analogous terms in the Kinetic Theory of Gases. In this theory, transport due to fluctuating molecular velocities is modeled by gradient diffusion. On a much weaker basis, this is also the approach for turbulent convection [11]. The "turbulent" diffusion coefficient $\Gamma\left(\left[\mathrm{m}^{2} \mathrm{~s}^{-1}\right]\right)$ should be chosen as to reflect the nature of the fluctuating velocity field. A good model should take into account the fact that as Re diminishes the flow becomes laminar. $\Gamma(R e)$ can be obtained for example from $k-\varepsilon$ models. The second open term, the molecular mixing (first term on the right hand side), will remain open for the moment. A simple closure will be given later in our derivation.

As it happens in all transport equations for averaged quantities in turbulent flows, there are two intrinsic length scales in Equation 2. The largest one is associated to the grid-size and defines the limit of the numerically solved length-scales, where the PDF varies smoothly. As a consequence, scalar averages (mean, variance,...), which are obtained by means of the PDF, will vary smoothly also at that length scale. Derivatives outside any kind of averaged quantities are then taken using this length scale. The other one is the Batchelor length scale which is of the range of the smallest scales in the scalar field, and for $\gamma$ of the order of the viscosity, is about the size of the Kolmogorov length scale. This scale affects the derivatives inside any kind of averages in Equation 2. The averaging operator \langle\rangle is responsible for smoothing the small scales into the big ones.

The resulting transport equation for the PDF with the turbulent convection already modeled 


$$
\frac{\partial P_{c}}{\partial t}+U_{i} \frac{\partial P_{c}}{\partial x_{i}}=-\frac{\partial^{2}}{\partial \phi^{2}}\left[\gamma\left\langle\frac{\partial c}{\partial x_{i}} \frac{\partial c}{\partial x_{i}} \mid c=\phi\right\rangle P_{c}\right]+\frac{\partial}{\partial x_{i}}\left[(\Gamma+\gamma) \frac{\partial P_{c}}{\partial x_{i}}\right]
$$

Having reached this point, it is convenient to briefly describe the Eulerian Monte Carlo method $(\mathrm{EMC})[2]$.

In order to solve Eq. 3, it is convenient to use a Monte Carlo approach. This is due to the high dimensionality (scalar, space, time) introduced by the PDF, which is worsened by the addition of more scalars to the formulation, as it typically occurs in turbulent combustion. Among Monte Carlo methods, the Eulerian approach has proved to be an excellent alternative to Lagrangian formulations. In that formulation, we want to represent $P_{c}$ by an ensemble of $N$ stochastic fields $\mathfrak{r}^{n}(\mathbf{x}, t)$, twice differentiable in space:

$$
P_{c}(\phi ; \mathbf{x}, t)=\langle\delta[\phi-\mathfrak{r}(\mathbf{x}, t)]\rangle \approx \frac{1}{N} \sum_{n=1}^{N} \delta\left[\phi-\mathfrak{r}^{n}(\mathbf{x}, t)\right]
$$

where $\delta$ represents the Dirac delta function. Those $N$ stochastic fields are not real values of the flow field (they can't be, as they are smooth at the big scales, while the real scalar field is smooth at the small scales), but form an equivalent stochastic system in the sense that $\mathfrak{r}(\mathbf{x}, t)$ and $c(\mathbf{x}, t)$ share the same one-point PDF.

Next, we proceed similarly as in the EMC introductory paper [2], but in slightly different way, more consistent with diffusion coefficients depending on space. Consider the diffusion term:

$$
\frac{\partial}{\partial x_{i}}\left[(\Gamma+\gamma) \frac{\partial P_{c}}{\partial x_{i}}\right]=\frac{\partial}{\partial x_{i}}\left[(\Gamma+\gamma) \frac{\partial\langle\delta[\phi-\mathfrak{r}(\mathbf{x}, t)]\rangle}{\partial x_{i}}\right]
$$

Taking into account that derivatives of Dirac's delta can be calculated by mains of the chain rule,

$$
\frac{\partial \delta[\phi-\mathfrak{r}(\mathbf{x}, t)]}{\partial x_{i}}=\frac{\partial \delta}{\partial \mathfrak{r}} \frac{\partial \mathfrak{r}}{\partial x_{i}}=-\frac{\partial \delta}{\partial \phi} \frac{\partial \mathfrak{r}}{\partial x_{i}}
$$

we substitute Eq. 4 into the right hand term of Eq. 5, without considering the averaging operator for convenience:

$$
\frac{\partial}{\partial x_{i}}\left[(\Gamma+\gamma) \frac{\partial \delta}{\partial x_{i}}\right]=-\frac{\partial}{\partial \phi}\left\{\delta \frac{\partial}{\partial x_{i}}\left[(\Gamma+\gamma) \frac{\partial \mathfrak{r}}{\partial x_{i}}\right]\right\}+\frac{\partial^{2}}{\partial \phi^{2}}\left[\delta(\Gamma+\gamma) \frac{\partial \mathfrak{r}}{\partial x_{i}} \frac{\partial \mathfrak{r}}{\partial x_{i}}\right]
$$


Recovering the averaging operator, the diffusion term is re-expressed as

$$
\frac{\partial}{\partial x_{i}}\left[(\Gamma+\gamma) \frac{\partial P_{c}}{\partial x_{i}}\right]=-\frac{\partial}{\partial \phi}\left\{\left\langle\frac{\partial}{\partial x_{i}}\left[(\Gamma+\gamma) \frac{\partial \mathfrak{r}}{\partial x_{i}}\right] \mid \mathfrak{r}=\phi\right\rangle P_{c}\right\}+\frac{\partial^{2}}{\partial \phi^{2}}\left[(\Gamma+\gamma)\left\langle\frac{\partial \mathfrak{r}}{\partial x_{i}} \frac{\partial \mathfrak{r}}{\partial x_{i}} \mid \mathfrak{r}=\phi\right\rangle P_{c}\right]
$$

The diffusion term is then replaced in Eq. 3 to get a convenient form for the PDF transport equation:

$$
\begin{aligned}
\frac{\partial P_{c}}{\partial t}+U_{i} \frac{\partial P_{c}}{\partial x_{i}} & =-\frac{\partial}{\partial \phi}\left\{\left\langle\frac{\partial}{\partial x_{i}}\left[(\Gamma+\gamma) \frac{\partial \mathfrak{r}}{\partial x_{i}}\right] \mid \mathfrak{r}=\phi\right\rangle P_{c}\right\} \\
& +\frac{\partial^{2}}{\partial \phi^{2}}\left[\Gamma\left\langle\frac{\partial \mathfrak{r}}{\partial x_{i}} \frac{\partial \mathfrak{r}}{\partial x_{i}} \mid \mathfrak{r}=\phi\right\rangle P_{c}\right] \\
& +\frac{\partial^{2}}{\partial \phi^{2}}\left[\gamma\left\langle\frac{\partial \mathfrak{r}}{\partial x_{i}} \frac{\partial \mathfrak{r}}{\partial x_{i}} \mid \mathfrak{r}=\phi\right\rangle P_{c}\right]-\frac{\partial^{2}}{\partial \phi^{2}}\left[\gamma\left\langle\frac{\partial c}{\partial x_{i}} \frac{\partial c}{\partial x_{i}} \mid c=\phi\right\rangle P_{c}\right] .
\end{aligned}
$$

At this point we will follow a slightly different approach to the one used in the original paper [2].

For high Reynolds numbers, we can make the following assumption for the terms in $\gamma$ at the third line of the previous equation, namely:

$$
\begin{aligned}
\frac{\partial^{2}}{\partial \phi^{2}}\left[\gamma\left\langle\frac{\partial \mathfrak{r}}{\partial x_{i}} \frac{\partial \mathfrak{r}}{\partial x_{i}} \mid \mathfrak{r}=\phi\right\rangle P_{c}\right] & -\frac{\partial^{2}}{\partial \phi^{2}}\left[\gamma\left\langle\frac{\partial c}{\partial x_{i}} \frac{\partial c}{\partial x_{i}} \mid c=\phi\right\rangle P_{c}\right] \\
& \approx-\frac{\partial}{\partial \phi^{2}}\left[\gamma\left\langle\frac{\partial c^{\prime}}{\partial x_{i}} \frac{\partial c^{\prime}}{\partial x_{i}} \mid c=\phi\right\rangle P_{c}\right],
\end{aligned}
$$

where $c$ has been split in its mean and fluctuating parts, $c=C+c^{\prime}$.

The reasons for the approximation 10 are next explained. Since the EMC fields are smooth at the resolved scales, their contribution (first term) is of the same order as the contribution of the mean gradients (second term), and approximately these terms cancel each other out against the contribution of the fluctuating gradients (third term) which is dominant. In fact the second term is already negligible (of the order of $R e^{-1}$ ) with respect to the third one [12].

But Eq. 10 is also true in the low Reynolds number limit. As $R e \rightarrow 0$, the fluctuations dissapear, so the first term becomes equal to the second term and they cancel each other out. The third term vanishes. This property assures that approximation 10 will not hinder the proper behavior of the EMC fields in the laminar limit. 
With approximation 10, Eq. 9 becomes:

$$
\begin{aligned}
\frac{\partial P_{c}}{\partial t}+U_{i} \frac{\partial P_{c}}{\partial x_{i}} & =-\frac{\partial}{\partial \phi}\left\{\left\langle\frac{\partial}{\partial x_{i}}\left[(\Gamma+\gamma) \frac{\partial \mathfrak{r}}{\partial x_{i}}\right] \mid \mathfrak{r}=\phi\right\rangle P_{c}\right\} \\
& +\frac{\partial^{2}}{\partial \phi^{2}}\left[\Gamma\left\langle\frac{\partial \mathfrak{r}}{\partial x_{i}} \frac{\partial \mathfrak{r}}{\partial x_{i}} \mid \mathfrak{r}=\phi\right\rangle P_{c}\right] \\
& -\frac{\partial^{2}}{\partial \phi^{2}}\left[\gamma\left\langle\frac{\partial c^{\prime}}{\partial x_{i}} \frac{\partial c^{\prime}}{\partial x_{i}} \mid c=\phi\right\rangle P_{c}\right]
\end{aligned}
$$

Now, we can proceed as Valiño [2] to obtain the evolution of the EMC fields, previously closing the micro-mixing contribution with a Linear Mean Square Estimation (LMSE) model [13], remembering that now micro-mixing is related just to the sub-grid part of the scalar dissipation. Of course, other micro-mixing closures, like the Langevin model [14], are possible. The resulting transport equations are, for a particular field $\mathfrak{r}^{n}$ and following Itô's approximation:

$$
\mathrm{d} \mathfrak{r}^{n}=-U_{i} \frac{\partial \mathfrak{r}^{n}}{\partial x_{i}} \mathrm{~d} t+\frac{\partial}{\partial x_{i}}\left[(\Gamma+\gamma) \frac{\partial \mathfrak{r}^{n}}{\partial x_{i}}\right] \mathrm{d} t+(2 \Gamma)^{1 / 2} \frac{\partial \mathfrak{r}^{n}}{\partial x_{i}} \mathrm{~d} W_{i}^{n}-\omega_{c^{\prime}} / 2\left(\mathfrak{r}^{n}-C\right) \mathrm{d} t
$$

where the scalar charactertic dissipation frequency is defined as $\omega_{c^{\prime}}=\frac{\left\langle\gamma \frac{\partial c^{\prime}}{\partial x_{i}} \frac{\partial c^{\prime}}{\partial x_{i}}\right\rangle}{\left\langle c^{2}\right\rangle / 2}$, which is to be provided by some other means [15]. This equation can now be compared with the one obtained in the original formulation [2]:

$$
\mathrm{d} \mathfrak{r}^{n}=-U_{i} \frac{\partial \mathfrak{r}^{n}}{\partial x_{i}} \mathrm{~d} t+\frac{\partial}{\partial x_{i}}\left[(\Gamma+\gamma) \frac{\partial \mathfrak{r}^{n}}{\partial x_{i}}\right] \mathrm{d} t+(2 \Gamma+2 \gamma)^{1 / 2} \frac{\partial \mathfrak{r}^{n}}{\partial x_{i}} \mathrm{~d} W_{i}^{n}-\omega_{c} / 2\left(\mathfrak{r}^{n}-C\right) \mathrm{d} t,
$$

where $\omega_{c^{\prime}}=\frac{\left\langle\gamma \frac{\partial c}{\partial x^{\prime}} \frac{\partial c}{\partial x_{i}}\right\rangle}{\left\langle c^{\prime 2}\right\rangle / 2}$. In the original formulation, in the laminar limit ( $\Gamma$ vanishes), the EMC fields keep being stochastic through the Wiener term in $\gamma$, the molecular diffusion. We obviously want the EMC fields to become non-stochastic when the turbulent diffusion coefficient $\Gamma$ tends to zero. In Eq. 12, the model does not have this spurious contribution. Another desirable property, just from a theoretical point of view, is that in the second formulation the dissipation characteristic frequency $\omega_{c^{\prime}}$ depends just on the fluctuating gradients. This spliting of micro-mixing contributions is analogous to the separation of dissipation of kinetic energy between mean and fluctuating parts used by Pope [12]. In fact Pope already considered molecular mixing models models restricted to the fluctuating dissipation. Indeed, if we keep the mean gradients in the molecular mixing closure, 
we are somehow "over-modeling" the micro-mixing. In compensation, the spurious Wiener term in $\gamma$ appears. These differences are not an issue at high Reynolds numbers, when fluctuating gradients are dominant and $\gamma<<\Gamma$, but make the new version of the model consistent in the laminar limit. In fact this consistency is guaranteed because the approximation 10 and the models used to close the PDF transport equation, gradient diffusion and LMSE excluding mean gradients, are valid in the laminar limit.

Observe that in the laminar case, with the new formulation, the EMC fields are all equal and follow exactly Eq. 1(zero variance). This is the correct behavior, which the original formulation was lacking. Again, conceptually, the basis to make it so lies on the splitting between mean gradient and fluctuating contributions in the molecular mixing term, which obviously makes a difference for low Reynolds numbers. In the original formulation, the micro-mixing term included the mean gradient contribution, so $\omega_{c}>\omega_{c^{\prime}}$. The extra dissipation is compensated by the Wiener term in $\gamma$. By removing the mean gradient contribution and using $\omega_{c^{\prime}}$, such compensation is not needed any more and the Wiener term disappears from the formulation, which results in Eq. 12 consistent at the laminar limit.

From a practical point of view, the difference when implementing the new approach with respect the old formulation will mostly appear at wall boundary conditions for the EMC fields. In the new formulation, the fields will not have any spurious stochastic noise added. This will obviously add stability to the solver.

Notice that in fact this formulation is also consistent with the low grid Reynolds number limit, that is, with the direct numerical simulation (DNS) limit. In such situation, the flow is still turbulent, but all the velocity and scalar scales are solved, so no subgrid transport or subrig molecular mixing exist, which means $\Gamma$ is zero and there is no LMSE (or alternative model). The standard Navier-Stokes equations are then recovered, as in the laminar limit. However, as the flow is turbulent and hence chaotic, a small difference in the boundary conditions or a small fluctuation (round-off errors) would make each EMC field different. They would be like different realizations of the same turbulent flow. Statistical averages could be obtained by ensemble averaging over 
those realizations.

It is well known that a gradient diffusion model is a correct approximation for fluctuating convection by "white noise" fluctuating velocity fields. In fact, the Stratonovich version of the EMC formulation is a consistent proof. In the old EMC formulation (Eq. 13), $\Gamma+\gamma$ appears like a unified coefficient in the second and third terms of the right hand side of Eq. 13 which is equivalent to say that the fluctuating advection by this velocity field is responsible of both turbulent convection and diffusion fluxes at grid level, to the point of claiming that Eq. 13, in its reacting version, is a pure advection equation with source terms [6]. This is obviously unphysical. Molecular diffusion should not show such behavior. In the new formulation, this inconsistency dissapears, as $\gamma$ does not appear in the Wiener term any more in Eq. 12, so the stochastic formulation keeps a proper diffusive contribution, in the sense explained.

In the derivation of the formulation, the velocity field has been considered solenoidal, that is, the density does not change with the scalar concentration. This approach has been considered for simplicity and to ease the comparison with the introductory paper of the EMC method. In principle, the present derivation is valid for variable density flows, as no explicit use of the solenoidal character of the velocity field has been done. However, velocity formulations for variable density reacting flows use Favre averaged velocities, and to match the velocity formulation, a Favre PDF transport equation should be used for scalars. The derivation does not present difficulties and it has been added in an appendix.

\section{Conclusions}

Reminding Durbin and Petterson's cite at the introduction that turbulent models are developed for fully turbulent conditions, however, most transport equation models do converge to a laminar solution. That was not the case for the original formulation of the EMC field method. In this paper, it has been presented an alternative and slightly different version of the EMC field method which is consistent in the laminar limit. The EMC fields evolve just following the ordinary convectiondiffusion equation in the laminar limit, without the spurious Wiener term in the scalar diffusion 
coefficient exibited in the original formulation. The key point is to separate mean gradient and fluctuating contributions in the micro-mixing term. Only the fluctuating part is left for modeling, which is more consistent with the idea of a model closure.

\section{Appendix}

The basics of the new version of the EMC field method are best introduced, for the sake of simplicity, in the case of a single scalar in a constant density turbulent flow. However the practical use of the EMC field method is for multiscalar reacting, density variable (low Mach) turbulent flows. In this appendix, EMC field transport equations for this general case are derived. Only the main ideas of this derivation are outlined here, as it is an almost straightforward extension of the single scalar constant density flow case.

The transport equation for a set of $Q$ scalars convected by a velocity field in a turbulent flow is

$$
\frac{\partial c_{\alpha}}{\partial t}+u_{j} \frac{\partial c_{\alpha}}{\partial x_{j}}=\frac{1}{\rho} \frac{\partial}{\partial x_{j}}\left(\gamma_{\rho} \frac{\partial c_{\alpha}}{\partial x_{j}}\right)+S_{\alpha}(\mathbf{c})
$$

where $u_{j}$ is the component $j$ of the velocity, $c_{\alpha}$ is the scalar $\alpha(\alpha=1, \ldots, Q)$ (one of them is usually the enthalpy), $\gamma_{\rho}$ is a diffusion coefficient $\left(\left[\mathrm{kg} \mathrm{m}^{-1} \mathrm{~s}^{-1}\right]\right.$ ) (in the constant density case we defined $\gamma=\frac{\gamma_{\rho}}{\rho}$ ), which is taken equal for all the scalars, as it is common in combustion for simplicity. Finally, $S_{\alpha}(\mathbf{c})$ is the source term of the scalar $\alpha$, coming from the chemical reactions (or heat exchange in the case of enthalpy). The density is a function of the scalars at the reference pressure (low Mach approximation). Then we have $\rho=\rho(\mathbf{c})$

For variable density flows, it is convenient to work with density-weighted averages, also known as Favre averages. For a quantity $\bullet$, its density-weighted average is defined bye

$$
\widetilde{\bullet} \equiv \frac{\langle\rho \bullet\rangle}{\langle\rho\rangle},
$$

One of the main reasons for the use of this formulation is that velocity Favre average verifies the continuity equation:

$$
\frac{\partial\langle\rho\rangle}{\partial t}+\frac{\partial}{\partial x_{j}}\left(\langle\rho\rangle \widetilde{u_{j}}\right)=0
$$


which is straightforward derived by averaging the instantaneous continuity equation. Some techniques, like laser doppler anemometry, take direct measurements of density-weighted magnitudes [16] The Eulerian density-weighted PDF is obtained by Favre averaging the fine grained PDF of the scalar (Dirac's delta):

$$
\widetilde{P}_{\mathbf{c}}(\phi ; \mathbf{x}, t)=\frac{\rho(\phi) P_{\mathbf{c}}(\phi ; \mathbf{x}, t)}{\langle\rho\rangle}
$$

This is the PDF to be used for calculating scalar density-weighted averages, as defined in Eq. 15. Its transport equation can be derived by the same standard means as the single scalar PDF (use of Eq 16 is needed):

$$
\begin{aligned}
\frac{\partial \widetilde{P}_{\mathbf{c}}}{\partial t} & +\widetilde{u}_{i} \frac{\partial \widetilde{P}_{\mathbf{c}}}{\partial x_{i}}+\frac{1}{\langle\rho\rangle} \frac{\partial}{\partial x_{i}}\left(\langle\rho\rangle\left\langle u^{\prime \prime}{ }_{i} \mid \mathbf{c}=\boldsymbol{\phi}\right\rangle \widetilde{P}_{\mathbf{c}}\right) \\
& =-\frac{1}{\langle\rho\rangle} \frac{\partial^{2}}{\partial \phi_{\alpha} \partial \phi_{\beta}}\left[\gamma_{\rho}\left\langle\frac{\partial c_{\alpha}}{\partial x_{i}} \frac{\partial c_{\beta}}{\partial x_{i}} \mid \mathbf{c}=\phi\right\rangle P_{c}\right]+\frac{1}{\langle\rho\rangle} \frac{\partial}{\partial x_{i}}\left(\gamma_{\rho} \frac{\partial P_{c}}{\partial x_{i}}\right)-\frac{\partial S_{\alpha}(\phi)}{\partial \phi_{a}}
\end{aligned}
$$

where $u_{i}^{\prime \prime}$ is the $i$ component of the fluctuating velocity with respect to the density-weighted velocity. The terms in $u_{i}^{\prime \prime}$ (turbulent transport) and in the cross scalar derivatives (molecular mixing) need a model. Those models are the natural extensions to the variable density multiscalar problem. For the turbulent transport, a gradient closure model is used:

$$
\left\langle u^{\prime \prime}{ }_{i} \mid \mathbf{c}=\phi\right\rangle \widetilde{P}_{\mathbf{c}} \approx-\frac{\Gamma_{\rho}}{\langle\rho\rangle} \frac{\partial \widetilde{P}_{\mathbf{c}}}{\partial x_{i}}
$$

where $\Gamma_{\rho}$ is a turbulent diffusion coefficient $\left(\left[\mathrm{kg} \mathrm{m}^{-1} \mathrm{~s}^{-1}\right]\right)$ (in the constant density case we defined $\left.\Gamma=\frac{\Gamma_{\rho}}{\rho}\right)$. Molecular mixing will be later modeled.

Replacing Eq. 19 in Eq. 18, the next equation is otained, which is the analogous to Eq. 3 for this multiscalar reacting case:

$$
\frac{\partial \widetilde{P}_{\mathbf{c}}}{\partial t}=-\frac{1}{\langle\rho\rangle} \frac{\partial^{2}}{\partial \phi_{\alpha} \partial \phi_{\beta}}\left[\gamma_{\rho}\left\langle\frac{\partial c_{\alpha}}{\partial x_{i}} \frac{\partial c_{\beta}}{\partial x_{i}} \mid \mathbf{c}=\phi\right\rangle P_{c}\right]+\frac{1}{\langle\rho\rangle} \frac{\partial}{\partial x_{i}}\left[\left(\Gamma_{\rho}+\gamma_{\rho}\right) \frac{\partial P_{c}}{\partial x_{i}}\right]
$$

Analogously to the single scalar case, we represent now $\widetilde{P}_{\mathbf{c}}$ by an ensemble of $N$ multiscalar stochastic fields, twice differentiable in space:

$$
\widetilde{P}_{\mathbf{c}}(\phi ; \mathbf{x}, t)=\langle\delta[\phi-\mathfrak{r}(\mathbf{x}, t)]\rangle \approx \frac{1}{N} \sum_{n=1}^{N} \prod_{\alpha=1}^{Q} \delta\left[\phi_{\alpha}-\mathfrak{r}_{\alpha}^{n}(\mathbf{x}, t)\right],
$$


where $\mathfrak{r}_{\alpha}^{n}(\mathbf{x}, t)$ is the scalar $\alpha$ of the field $n$.

By expanding in Eq. 20 the PDF diffusive term, as in Eq. 5, the Favre multiscalar version of Eq 9 is obtained:

$$
\begin{aligned}
\frac{\partial \widetilde{P}_{\mathbf{c}}}{\partial t}+\widetilde{u}_{i} \frac{\partial \widetilde{P}_{\mathbf{c}}}{\partial x_{i}} & =-\frac{1}{\langle\rho\rangle} \frac{\partial}{\partial \phi_{\alpha}}\left\{\left\langle\frac{\partial}{\partial x_{i}}\left[\left(\Gamma_{\rho}+\gamma_{\rho}\right) \frac{\partial \mathfrak{r}_{\alpha}}{\partial x_{i}}\right] \mid \mathfrak{r}=\phi\right\rangle \widetilde{P}_{\mathbf{c}}\right\} \\
& +\frac{1}{\langle\rho\rangle} \frac{\partial^{2}}{\partial \phi_{\alpha} \partial \phi_{\beta}}\left[\Gamma_{\rho}\left\langle\frac{\partial \mathfrak{r}_{\alpha}}{\partial x_{i}} \frac{\partial \mathfrak{r}_{\beta}}{\partial x_{i}} \mid \mathfrak{r}=\phi\right\rangle \widetilde{P}_{\mathbf{c}}\right]+\frac{1}{\langle\rho\rangle} \frac{\partial^{2}}{\partial \phi_{\alpha} \partial \phi_{\beta}}\left[\gamma_{\rho}\left\langle\frac{\partial \mathfrak{r}_{\alpha}}{\partial x_{i}} \frac{\partial \mathfrak{r}_{\beta}}{\partial x_{i}} \mid \mathfrak{r}=\boldsymbol{\phi}\right\rangle \widetilde{P}_{\mathbf{c}}\right] \\
& -\frac{1}{\langle\rho\rangle} \frac{\partial^{2}}{\partial \phi_{\alpha} \partial \phi_{\beta}}\left[\gamma_{\rho}\left\langle\frac{\partial c_{\alpha}}{\partial x_{i}} \frac{\partial c_{\beta}}{\partial x_{i}} \mid \mathbf{c}=\phi\right\rangle \widetilde{P}_{\mathbf{c}}\right]-\frac{\partial S_{\alpha}(\phi)}{\partial \phi_{a}} .
\end{aligned}
$$

Approximation 10 takes the following form in the multiscalar case:

$$
\begin{aligned}
\frac{\partial^{2}}{\partial \phi_{\alpha} \partial \phi_{\beta}}\left[\gamma_{\rho}\left\langle\frac{\partial \mathfrak{r}_{\alpha}}{\partial x_{i}} \frac{\partial \mathfrak{r}_{\beta}}{\partial x_{i}} \mid \mathfrak{r}=\phi\right\rangle \widetilde{P}_{\mathbf{c}}\right] & -\frac{\partial^{2}}{\partial \phi_{\alpha} \partial \phi_{\beta}}\left[\gamma_{\rho}\left\langle\frac{\partial c_{\alpha}}{\partial x_{i}} \frac{\partial c_{\beta}}{\partial x_{i}} \mid \mathbf{c}=\phi\right\rangle \widetilde{P}_{\mathbf{c}}\right] \\
& \approx-\frac{\partial^{2}}{\partial \phi_{\alpha} \partial \phi_{\beta}}\left[\gamma_{\rho}\left\langle\frac{\partial c_{\alpha}^{\prime}}{\partial x_{i}} \frac{\partial c_{\beta}^{\prime}}{\partial x_{i}} \mid \mathbf{c}=\phi\right\rangle \widetilde{P}_{\mathbf{c}}\right]
\end{aligned}
$$

whis is valid both for high Reynolds and in the laminar limit. Using this approximation to replace the third and fourth terms of the right hand side of Eq. 23 results in:

$$
\begin{aligned}
\frac{\partial \widetilde{P}_{\mathbf{c}}}{\partial t}+\widetilde{u}_{i} \frac{\partial \widetilde{P}_{\mathbf{c}}}{\partial x_{i}} & =-\frac{1}{\langle\rho\rangle} \frac{\partial}{\partial \phi_{\alpha}}\left\{\left\langle\frac{\partial}{\partial x_{i}}\left[\left(\Gamma_{\rho}+\gamma_{\rho}\right) \frac{\partial \mathfrak{r}_{\alpha}}{\partial x_{i}}\right] \mid \mathfrak{r}=\phi\right\rangle \widetilde{P}_{\mathbf{c}}\right\} \\
& +\frac{1}{\langle\rho\rangle} \frac{\partial^{2}}{\partial \phi_{\alpha} \partial \phi_{\beta}}\left[\Gamma_{\rho}\left\langle\frac{\partial \mathfrak{r}_{\alpha}}{\partial x_{i}} \frac{\partial \mathfrak{r}_{\beta}}{\partial x_{i}} \mid \mathfrak{r}=\phi\right\rangle \widetilde{P}_{\mathbf{c}}\right] \\
& -\frac{1}{\langle\rho\rangle} \frac{\partial^{2}}{\partial \phi_{\alpha} \partial \phi_{\beta}}\left[\gamma_{\rho}\left\langle\frac{\partial c_{\alpha}^{\prime}}{\partial x_{i}} \frac{\partial c_{\beta}^{\prime}}{\partial x_{i}} \mid \mathbf{c}=\phi\right\rangle \widetilde{P}_{\mathbf{c}}\right]-\frac{\partial S_{\alpha}(\phi)}{\partial \phi_{a}}
\end{aligned}
$$

Finally, we close the molecular mixing term using LMSE again:

$$
\frac{1}{\langle\rho\rangle} \frac{\partial^{2}}{\partial \phi_{\alpha} \partial \phi_{\beta}}\left[\gamma_{\rho}\left\langle\frac{\partial c_{\alpha}^{\prime}}{\partial x_{i}} \frac{\partial c_{\beta}^{\prime}}{\partial x_{i}} \mid \mathbf{c}=\phi\right\rangle \widetilde{P}_{\mathbf{c}}\right] \approx \frac{\partial}{\partial \phi_{\alpha}}\left[\omega_{c_{[\alpha]}^{\prime}}\left(\phi_{\alpha}-C_{\alpha}\right) \widetilde{P}_{\mathbf{c}}\right]
$$

where $\omega_{c_{[\alpha]}^{\prime}}^{\prime}=\frac{\left\langle\frac{\gamma \rho}{\rho} \frac{\partial c_{[\alpha]}^{\prime}}{\partial x_{i}} \frac{\partial c_{[\alpha]}^{\prime}}{\partial x_{i}}\right\rangle}{\left\langle c_{[\alpha]}^{\prime}\right\rangle / 2}$ is the characteristic dissipation frequency for scalar $\alpha$ (no sum in $\alpha$ is indicated by $[\alpha]$ ). The closed PDF transport equation is then, moving $\langle\rho\rangle$ inside $\phi$ derivatives for convenience:

$$
\begin{aligned}
\frac{\partial \widetilde{P}_{\mathbf{c}}}{\partial t}+\widetilde{u}_{i} \frac{\partial \widetilde{P}_{\mathbf{c}}}{\partial x_{i}} & =-\frac{\partial}{\partial \phi_{\alpha}}\left\{\frac{1}{\langle\rho\rangle}\left\langle\frac{\partial}{\partial x_{i}}\left[\left(\Gamma_{\rho}+\gamma_{\rho}\right) \frac{\partial \mathfrak{r}_{\alpha}}{\partial x_{i}}\right] \mid \mathfrak{r}=\phi\right\rangle \widetilde{P}_{\mathbf{c}}\right\} \\
& +\frac{\partial^{2}}{\partial \phi_{\alpha} \partial \phi_{\beta}}\left[\frac{\Gamma_{\rho}}{\langle\rho\rangle}\left\langle\frac{\partial \mathfrak{r}_{\alpha}}{\partial x_{i}} \frac{\partial \mathfrak{r}_{\beta}}{\partial x_{i}} \mid \mathfrak{r}=\phi\right\rangle \widetilde{P}_{\mathbf{c}}\right] \\
& -\frac{\partial}{\partial \phi_{\alpha}}\left[\omega_{c_{[\alpha]}^{\prime}}\left(\phi_{\alpha}-C_{\alpha}\right) \widetilde{P}_{\mathbf{c}}\right]-\frac{\partial S_{\alpha}(\phi)}{\partial \phi_{a}}
\end{aligned}
$$


The resulting transport equation for a particular field scalar $\mathfrak{r}_{\alpha}^{n}$ is, following Itô's approximation:

$\mathrm{d} \mathfrak{r}_{\alpha}^{n}=-\widetilde{u}_{i} \frac{\partial \mathfrak{r}_{\alpha}^{n}}{\partial x_{i}} \mathrm{~d} t+\frac{1}{\langle\rho\rangle} \frac{\partial}{\partial x_{i}}\left[\left(\Gamma_{\rho}+\gamma_{\rho}\right) \frac{\partial \mathfrak{r}_{\alpha}^{n}}{\partial x_{i}}\right] \mathrm{d} t+\left(2 \frac{\Gamma_{\rho}}{\langle\rho\rangle}\right)^{1 / 2} \frac{\partial \mathfrak{r}_{\alpha}^{n}}{\partial x_{i}} \mathrm{~d} W_{i}^{n}-\omega_{c_{[\alpha]}^{\prime}} / 2\left(\mathfrak{r}_{\alpha}^{n}-C_{\alpha}\right) \mathrm{d} t+S_{\alpha}\left(\mathfrak{r}^{n}\right) \mathrm{d} t$

A comment is pertinent here about the Wiener process in Eq. 27. Physically, all the scalars are convected together by the turbulent transport, so this term cannot induce decorrelation between any two scalars in the flow. That means that the same Wiener process is shared by all the scalars in the prevous equation. This is equivalent to say that a properly closed PDF turbulent transport equation should not contain cross derivatives in $\alpha, \beta$ in $\Gamma_{\rho}$ terms. Only the "diagonal" contribution $(\alpha=\beta)$ is to be kept. This is not true for molecular mixing, which physically induce decorrelation between scalars. Notice, however that for the particular case of LMSE closure, this effect is ignored. Langevin kind of models are more sophisticated and can incorporate decorrelation effects, which reflects in cross derivatives in $\alpha, \beta$ appearing in the corresponding PDF terms.

Finally, in the laminar limit, both turbulent transport and molecular mixing vanish, so Eq. 27 takes the proper shape:

$$
\mathrm{d} \mathfrak{r}_{\alpha}^{n}=-u_{i} \frac{\partial \mathfrak{r}_{\alpha}^{n}}{\partial x_{i}} \mathrm{~d} t+\frac{1}{\rho\left(\mathfrak{r}^{n}\right)} \frac{\partial}{\partial x_{i}}\left(\gamma_{\rho} \frac{\partial \mathfrak{r}_{\alpha}^{n}}{\partial x_{i}}\right) \mathrm{d} t+S_{\alpha}\left(\mathfrak{r}^{n}\right) \mathrm{d} t
$$

where both mean velocity and density have been replaced by their laminar value.

\section{References}

[1] T. S. Lundgren. Distribution functions in the statistical theory of turbulence. Physics of Fluids, 10:969-975, 1967.

[2] Luis Valiño. A field monte carlo formulation for calculating the probability density function of a single scalar in a turbulent flow. Flow, Turbulence and Combustion, 60:157-172, 1998.

[3] R. Mustata, L. Valiño, C. Jiménez, W.P. Jones, and S. Bondi. A probability density function eulerian monte carlo field method for large-eddy simulations. application to a turbulent piloted methane/air diffusion flame (sandia d). Combustion and Flame, 145:88-104, 2006. 
[4] I. A. Dodoulas and S. Navarro-Martinez. Large eddy simulation of premixed turbulent flames using the probability density function approach. Flow, Turbulence and Combustion, 90(3):645-678, 2013.

[5] E. Loth. Progress in probability density function methods for turbulent reacting flows. Progress in Energy and Combustion Science, 36:168-259, 2010.

[6] O. Soulard and V. A. Sabelnikov. Rapidly decorrelating velocity-field model as a tool for solving one-point fokker-planequations for probability density functions of turbulent reactive scalars. Physical Review E, 72:016301, 1-20, 2005.

[7] W. P. Jones and B. E. Launder. The prediction of laminarization with a two-equation model. International Journal of Heat and Mass Transfer, 15:301-314, 1972.

[8] P. A. Durbin and B. A. Petterson Reif. Statistical theory and modeling for turbulent flows. John Wiley and sons, 2001. ISBN 0-471-49744-4.

[9] Luis Valiño and Juan Hierro. Boundary conditions for probability density function transport equations in fluid mechanics. Phys. Rev. E, 67(046310):1-7, 2003.

[10] César Dopazo. Probabilistic approach to tubulent mixing of a reactive scalar. Bulletin of American meteorological society, 57(5):637, 1976.

[11] Uriel Frisch. Turbulence. Cambridge University Press, 1995. ISBN 0-521-45713-0.

[12] S.B. Pope. Turbulent flows. Cambridge University Press, 2000. Pages 123-125.

[13] César Dopazo. Probability density function approach for an axisymmetric heated jet: centerline evolution. Physics of Fluids, 18:397, 1975.

[14] Luis Valiño and César Dopazo. A binomial langevin model for turbulent mixing. Physics of Fluids A, 3(13):3034-3037, 1991. 
[15] César Dopazo. Recent developments in PDF methods. In P. A. Libby and F. A. Williams, editors, Turbulent reactive flows, chapter 7, pages 375-474. Academic Press, San Diego, 1994. ISBN 0-12-447945-6.

[16] M. Mustafa Kamal, Ruigang Zhou, Saravanan Balusamy, and Simone Hochgreb. Favreand reynolds-averaged velocity measurements: interpreting piv and lda measurements in combustion. In Proceedings of the Combustion Institute, Volume 35, Issue 3, 2015, Pages 3803-3811, 2015. 